\title{
Fabian Freyenhagen*
}

\section{Dogmatischer Dogmatismusvorwurf}

\author{
Eine Replik auf Stefan Müller-Doohm und Roman Yos
}

https://doi.org/10.1515/dzph-2019-0003

\begin{abstract}
Does theorising always presuppose a programme of justification? Does the Critical Theory of Adorno and Horkheimer do so? Do they claim it does? The answer should be a resounding 'no' to all three question. In regard to the second and third question, I had sketched an argument to that effect in an earlier paper in this journal. In this paper, I offer a rejoinder to the critical reply offered by Stefan Müller-Doohm und Roman Yos on behalf of the Habermasian mainstream in Frankfurt School Critical Theory. This rejoinder depends on giving a negative answer also to the first question. In rejecting the Habermasian idea of a programme of justification, I stand accused of dogmatism and, following on from this, decisionism (for how else than by an arbitrary decision can one choose among dogmas?). I reveal that this accusation itself betrays a certain dogmatism - insofar as it accepts that such a programme of justification is undeniably possible and required, without consideration of evidence or arguments to the contrary. Self-reflective and critical theorising about society can, indeed must, take other forms.\#
\end{abstract}

Keywords: programme of justification, Frankfurt School, Critical Theory, T. W. Adorno, Max Horkheimer, Jürgen Habermas, dogmatism

*Kontakt: Fabian Freyenhagen, $\forall$ iniversity of Essex, School of Philosophy and Art History, Wivenhoe Park, Colchester CO4 3SQ, Vereinigtes Königreich; ffrey@essex.ac.uk 
Und dann kommen noch die angestellten Philosophen und machen uns zum Vorwurf, daß wir keinen festen Standpunkt hätten. ${ }^{1}$

Die These, dass Kritische Theorie als orthodox konzipiert (und betrieben) werden soll, ${ }^{2}$ war natürlich als Provokation gemeint. Orthodoxie war dabei in dreifacher Hinsicht im Spiel:

1. als Rückkehr zu den Anfängen der Frankfurter Schule (insbesondere zu Horkheimers Konzeption der Kritischen Theorie von 1937 bei gleichzeitiger Ablehnung von Habermas' begründungsprogrammatischer Kehrtwende der späten 1960er und der folgenden Jahrzehnte);

2. als Reflexion darüber, was essentiell für Kritische Theorie ist (eben nicht - so lautete meine Argumentation - das von Habermas und anderen geforderte und versuchte Begründungsprogramm);

3. und als Anerkennung davon, dass mit Kritischer Theorie in einem gewissen Sinne Strenggläubigkeit einhergeht (nämlich so, dass sie jenseits des Begründungsprogramms, in dem Interesse an Emanzipation zu verorten ist).

Das war leicht misszuverstehen. Die drei Punkte waren eng miteinander verknüpft, und Orthodoxie kann auch noch anders verstanden werden, war aber nur in diesen dreifachen Sinn gemeint. Orthodoxie im Sinne der vorherrschenden Meinung wurde ja gerade abgelehnt (mein Vorschlag einer zweiten Kehrtwende war in diesem Sinne heterodox). Und es ging auch nicht um Orthodoxie im Sinne vom starren, unkritischen Festhalten an Theoriegebilden (oder „heiligen Büchern“), die trotz veränderten historischen Realitäten unbedacht angewendet werden müssten.

Die Provokation ist insofern geglückt, als dass sie zur Diskussion angeregt hat. Müller-Doohm und Yos haben in dieser Zeitschrift eine kritische Replik vorgelegt und sich dabei nicht zurückgehalten: Sie werfen mir Dogmatismus, Dezisionismus, Undifferenziertheit, einen vagen Vernunftmaßstab, Hypostasierung, gar Kanonisierung und Zurechtlegung der Texte vor. Ich freue mich über die Gelegenheit zu antworten. Meine Argumentationsstrategie dabei ist es, den Spieß umzudrehen - kurz gesagt: Ich will im Folgenden nahelegen, dass der gegen meinen Ansatz gemachte Dogmatismusvorwurf den Kern der Einwände von Müller-Doohm und Yos darstellt, dieser Vorwurf aber selber dogmatisch vorgetragen wird.

1 Adorno (1997a), 83.

2 Freyenhagen (2017). 
Ich werde als erstes rekonstruieren, worin der Dogmatismusvorwurf gegen meine Neuorientierung der Kritischen Theorie (als Rückbesinnung auf die erste Generation der Frankfurter Schule) besteht, und auf diesen Vorwurf antworten (I). Dies ist, so hoffe ich, über die internen Debatten in der Kritischen Theorie hinaus von Interesse. Dann werde ich die von Müller-Doohm und Yos in Anschlag gebrachte Lesart von Horkheimers und Adornos Schriften kritisieren (II), bevor ich auf andere von Müller-Doohm und Yos gemachte Einwände kurz eingehe (III).

\section{Eine kleine Kritik des Dogmatismusvorwurfs}

Müller-Doohm und Yos rekonstruieren meine Ausführungen ganz richtig, wenn sie schreiben, dass mir zufolge Kritische Theorie auf ein Begründungsprogramm verzichten könne. ${ }^{3}$ Zwei andere, noch wichtigere Aspekte finden jedoch keine Erwähnung: meines Erachtens erübrigt sich ein Begründungsprogramm nicht nur, sondern es ist, erstens, letztlich unmöglich; ja, zweitens, einzufordern eines zu liefern, ist sogar moralisch oder ethisch problematisch („Frevel“ in Adornos provokanten Formulierung). ${ }^{4}$

Es ist dienlich, sich erst einmal in Erinnerung zu rufen, was ich mit „Begründungsprogramm“ meine:

Ich werde hier keine notwendigen und hinreichenden Bedingungen dafür ausführen, was mit „Begründungsprogramm“ gemeint ist - ich vermute, dass dies ohnehin ein unmögliches Unterfangen wäre. Es ist allerdings nicht unmöglich, eine hinweisende Definition anzubieten. Was mir vorschwebt ist das Unternehmen, was Christine Korsgaard versucht, wenn sie nach den Quellen der Normativität fragt, und wonach Jürgen Habermas und andere verlangen, wenn sie Theodor W. Adorno und Max Horkheimer dafür kritisieren, dass es deren Theorie an normativen Grundlagen fehle. Wie ich im Haupttext andeute, gibt es eine beeindruckende Vielfalt an jenen, die sich um ein Begründungsprogramm bemühen oder ein solches verlangen, mit teilweise ähnlichen Anliegen und Herangehensweisen und teilweise sehr verschiedenen. Es geht mir hier darum, dass es möglich ist, mit dem Begründungsprogramm in all seiner Vielfalt zu brechen, und dass Kritische Theorie damit brechen sollte. ${ }^{5}$

Weiterhin ist es hilfreich, klar zu sagen, dass die Ablehnung von Begründungsprogrammen nicht bedeutet, dass ich jegliche Form der Begründung, der Ver-

3 Müller-Doohm/Yos (2018), 2 (im Folgenden zit. als Rpl); vgl. auch ebd., 3.

4 Vgl. Freyenhagen (2017), 462 u. 463.

5 Ebd., 457, Fn. 2. 
nunft oder Theorie ablehne. ${ }^{6}$ Mein Entwurf schließt ja Selbstbesinnung, theoriegeleitete Reflexion, genealogische Selbstbefragung explizit ein - also Formen situierter theoretischer Anstrengung, die dennoch nicht Begründungsprogramm sind. Wenn ich mich, beispielsweise, angesichts einer Unrechtserfahrung oder eines Leids kritisch auflehne, muss ich die Erfahrung von Leid oder Unrecht möglichst genau einkreisen und bestimmen, sie von verwandten, aber anders gearteten Fällen unterscheiden. Ich kann veranlasst sein, darüber zu reflektieren, was genau diese Erfahrung so besonders problematisch macht, und darauf verwiesen sein, dafür genealogische Studien anzustellen (u. a., um zu prüfen, ob das Problematische an mir liegt - meiner Projektion oder meinem Ressentiment - statt der Welt) oder die unterliegende Sozialdynamik und ihre Genese zu analysieren. All dies ist geleitete theoretische Anstrengung, kann sogar systematischen Charakter annehmen, aber es muss nicht notwendigerweise in ein Begründungsprogramm führen oder ein solches voraussetzen. Ähnlich in Fragen der Interpretation: Wenn ich beispielsweise einem Autor eine bestimmte These zuschreibe, dann obliegt es mir, dafür textliche Belege zu liefern (und somit meine Interpretation zu ,begründen“). Dies anzuerkennen, ist durchaus kompatibel mit der Ablehnung der Möglichkeit (und Notwendigkeit) eines Begründungsprogramms im oben genannten Sinne. Ebenso kann ich Gründe vorbringen, um anderen näherzubringen, dass ein Begründungsprogramm letztlich unmöglich ist; damit biete ich aber selbst kein Begründungsprogramm an und muss es auch nicht. (Wichtig ist hier, dass ich von „Näherbringen“ spreche, nicht von zweifelsfreiem Beweisen, nicht einmal von Überzeugen.)

Die Gründe, die ich diesbezüglich anführte, sind von dreierlei Art. Verteidigern von Begründungsprogrammen geht es typischerweise darum, ,die (universalen) Bedingungen der Möglichkeit von Kritik“ abzusichern, ${ }^{7}$ und zwar auf eine Weise, die allgemein zustimmungsfähig ist, d. h. ungeachtet der jeweiligen Position in der sozio-ökonomischen Ordnung, der je eigenen Präferenzen und Lebenspläne sowie der historischen Situierung. In Müller-Doohms und Yos’ Referat des frühen Habermas soll dies beispielsweise durch eine rationale Rekonstruktion des emanzipatorischen Erkenntnisinteresses erzielt werden. ${ }^{8}$ Mit Horkheimer habe ich dagegen erstens eingewandt, dass wir uns nicht aus unserer historischen Situierung befreien können, so dass eine Absicherung dieser Art unmöglich ist. ${ }^{9}$ (Dass eine solche verlangt wird, zeigt sich in der Replik beispielsweise daran,

6 Vgl. ebd. (2017), 464.

7 Rpl, 10.

8 Rpl, 9-10.

9 Freyenhagen (2017), 459. 
dass sich Müller-Dohm und Yos ohne Rückgriff auf übergeschichtliche Subjekte oder Reaktionsweisen keine „Absicherung“ des emanzipatorischen Interesses denken können. ${ }^{10}$ ) Zweitens habe ich mit Horkheimer daraufhin gewiesen, dass die Neutralität - oder vielleicht besser formuliert: die Unparteilichkeit -, die für die verlangte Art der Absicherung notwendig ist, ebenfalls unerreichbar ist. ${ }^{11}$ Ich stelle Begründungsprogramme also nicht unter Neutralitätsverdacht, ${ }^{12}$ sondern kritisiere sie immanent dafür, dass sie ihrem eigenen Anspruch auf Neutralität nicht genügen. Drittens habe ich zugleich mit und gegen Honneth argumentiert, dass allgemeine Prinzipien und Werte $\mathrm{zu}$ leer sind, um wirkliche normative Arbeit zu leisten ${ }^{13}$ - mit Honneth, weil er dies Forst gegenüber in Anschlag bringt; gegen Honneth, weil er es mit Blick auf seine eigenen Vorschläge für Kriterien des moralischen Fortschritts zu vergessen scheint. ${ }^{14}$

Jedes einzelne dieser skizzierten Argumente gegen die Möglichkeit des angestrebten Begründungsprogramms kann natürlich angefochten werden, aber dies bedarf speziell auf dieses Thema kalibrierter (und überzeugender) Gegenargumente - und Müller-Doohm und Yos liefern derer keine. Ohne derartige Gegenargumente bleibt meine These, dass das geforderte Begründungsprogramm unmöglich ist, unangefochten. Und wenn es unmöglich ist, dann folgt wiederum, dass die Absicherung, die die Verfechter des Begründungsprogramms anstreben, nicht sinnvoll als das Gegenteil von Dogmatismus, Dezisionismus, Voluntarismus oder Beliebigkeit verstanden werden kann. Die Einwände von Müller-Doohm und Yos setzen nun aber gerade voraus, dass es dieses Gegenteil ist, denn ihre Argumentation lässt sich wie folgt rekonstruieren:

1. [Implizite These A:] Ein Begründungsprogramm der normativen Maßstäbe von Kritik ist möglich.

2. [Implizite These B:] Ein solches Begründungsprogramm ist notwendig.

3. [Implizite These C:] Jenseits eines Begründungsprogramm gibt es dogmatisch behauptete Positionen, zwischen denen nur voluntaristisch, ja beliebig gewählt werden kann (was auf Dezisionismus hinausläuft).

4. Folgte Kritische Theorie dem Vorschlag Freyenhagens und verzichtete auf ein Begründungsprogramm, geriete sie auf die schiefe Bahn des Dezisionis-

\section{$10 \mathrm{Rpl}, 3$.}

11 Freyenhagen (2017), 460-461.

$12 \mathrm{Rpl}, 13$.

13 Freyenhagen (2017), 462-463; vgl. ders. (2015) sowie Schaub (2015), 117-118.

14 Viertens habe ich mit Adorno argumentiert, dass es sich bei der Forderung, unsere moralischen Reaktionen durch ein Begründungsprogramm abzusichern, um einen „Frevel“ handelt, da diese Forderung das Wichtigste verkennt (Freyenhagen 2017, 463; vgl. ders. 2013a, insbes. Kap. 5 u. 7). Diesen Aspekt werde ich hier nicht weiter verfolgen. 
mus (oder zumindest drohte sie auf diese zu geraten, was nicht der Fall wäre, wenn nicht auf ein Begründungsprogramm verzichtet würde).

Die obigen Argumentationsskizzen sprechen gegen 1, was wiederum gegen 2 spricht. ${ }^{15}$ Damit lassen sich Dogmatismus, Voluntarismus, Beliebigkeit und Dezisionismus auch nicht mehr einfach als Gegenteil des Begründungsprogramms verstehen. Dogmatismus (etc.) müsste also anders verstanden werden, womit 3 in Frage steht und damit auch 4.

Im Duden wird „Dogmatismus“ als „starres, unkritisches Festhalten an Anschauungen, Lehrmeinungen o. Ä.“ definiert und „dogmatisch“ als „in Dogmatismus befangen; unkritisch denkend“. Ich habe gegen diese Definitionen an sich nichts einzuwenden. Nur wenn „unkritisch“ einfach mit Verzicht auf und/oder Ablehnung eines Begründungsprogramms gleichgesetzt wird, geht etwas schief. Denn dieser Verzicht und diese Ablehnung sind ja nicht einfach unbedacht, starr, beliebig - und folglich nicht notwendig unkritisch. Und sie lassen durchaus eine Unterscheidung zwischen kritisch und unkritisch $\mathrm{zu}$ - allerdings ist diese Unterscheidung dann nicht so abgesichert, wie es sich BegründungstheoretikerInnen erträumen. Anders gesagt: Selbst wenn es weder möglich noch notwendig ist, ein Begründungsprogramm anzubieten, gibt es Unterschiede zwischen einem unkritischem Festhalten an Anschauungen und Lehrmeinungen und einem kritischem Festhalten an denselben. In meinem Aufsatz spreche ich explizit von „Strategien der Selbstreflexion, Distanzierung (u. a. durch genealogische Studien) und sogar Selbstironie, der Einbeziehung von und Bezugnahme auf die Stimmen, die ausgeschlossen wurden und werden“; ${ }^{16}$ und die Nichtanwendung (oder nur minimale Anwendung) dieser Strategien kann als ein Merkmal eines unkritischen Festhaltens dienen. Verfechter des Begründungsprogramms - unmöglich, wie es nach meiner Argumentation ist - können nichts darüber hinaus anbieten; aber ohne es sind wir deshalb nicht einfach mittel- und machtlos angesichts von Irrationalismus, Dogmatismus oder Dezisionismus. (Ich komme in den Teilen II und III noch auf Dezisionismus zurück.)

15 Man könnte meinen, dass selbst wenn es unmöglich ist, ein Begründungsprogramm der normativen Maßstäbe der Kritik zu liefern, die Notwendigkeit dafür weiterbesteht - wie etwa Luther meint, dass Menschen durch den Umstand, dass es für sie unmöglich ist, nicht zu sündigen, nicht von der Verpflichtung, nicht zu sündigen, freigesprochen werden (vgl. Martin 2009). Aber dies scheint mir nicht auf Müller-Doohm und Yos zuzutreffen - bei ihnen scheint die Möglichkeit eines Begründungsprogramms als Bedingung für seine Notwendigkeit zu fungieren, und daher spricht meine Argumentation gegen diese Möglichkeit in ihrem Fall auch gegen dessen Notwendigkeit.

16 Freyenhagen (2017), 465; vgl. ders. (2013b). 
Im Folgenden biete ich eine Fallstudie an, die zeigen soll, was es bedeutet, dogmatisch mit Texten umzugehen. Dass es auch anders geht, ohne sich gleich der Idee (und Notwendigkeit) eines Begründungsprogramms zu verschreiben, sollte dabei ebenfalls deutlich werden.

\section{Von wegen Zurechtlegen}

Wenn Interpretationen zur Debatte stehen, gehört typischerweise zweierlei dazu, diese Debatte voranzubringen: (1) Die von der Gegenseite angebrachten Textstellen werden erfolgreich anders interpretiert; und (2) es gibt weitere Textstellen, die die vorgeschlagene (alternative) Lesart stützen. Müller-Doohm und Yos beschränken sich fast ausschließlich auf die zweite Vorgehensweise. Bevor ich mich ihren Textstellen zuwende, sollte noch angemerkt werden, dass der Goldstandard in einer solchen Debatte darin besteht, die für die eigene Interpretation ausschlaggebenden Begriffe und Ansichten explizit in den relevanten Texten vorzufinden. Manchmal ist es nicht möglich, diesem Goldstandard zu genügen - beispielsweise, wenn die relevanten Texte mit Blick auf den umstritten Sachverhalt einfach nicht hinreichend explizit sind -, aber es wiegt schwer, wenn es einer, aber nicht der anderen Interpretation gelingt, diesen Standard zu erfüllen.

Müller-Doohm und Yos werfen mir vor, dass ich mir Horkheimers programmatischen Text „Traditionelle und kritische Theorie“ in bestimmter Weise „zurechtleg[e]“ und ihn „eigenwillig“ lese. ${ }^{17}$ Darüber hinaus werfen sie mir vor, zu vermeiden, eigens auf die Dialektik der Aufklärung einzugehen, ein ,zentrales Werk“, von dem sie behaupten, dass es als „ein Begründungsprogramm gelesen werden muss, ohne dass das Projekt Kritische Theorie zusammenfallen würde wie ein Kartenhaus“. ${ }^{18}$ Und auch Adornos Negative Dialektik wird bemüht, da auch diese Schrift „,begründungstheoretisch“ zu verstehen sei. ${ }^{19}$

Zunächst möchte ich feststellen, dass der Begriff der „Begründung“ in nur einer der von Müller-Dohm und Yos vorgebrachten Textstellen überhaupt explizit vorkommt - und zwar in derjenigen Textstelle, die ich in meinem Artikel angeführt hatte. ${ }^{20}$ An dieser Stelle stellt Adorno eindeutig fest, dass der neue kategorische Imperativ ,widerspenstig gegen seine Begründung“ sei. ${ }^{21}$

17 Rpl, 3 u. 4.

$18 \mathrm{Rpl}, 6$; meine Hervorh.

$19 \mathrm{Rpl}, 8$.

20 Freyenhagen (2017), 462.

21 Adorno (1997b), 358 (im Folgenden zit. als ND). 
Anstatt auf Textstellen zu verweisen, in denen sich Horkheimer und/oder Adorno explizit oder zumindest eindeutig für ein Begründungsprogramm aussprechen, werden alle möglichen Formulierungen angeführt, die ein Bekenntnis zu einem solchen Programm keineswegs implizieren. In Bezug auf die Dialektik der Aufklärung handelt es sich bei dem mobilisierten Schlüsselbegriff um „Selbstbesinnung“; im Hinblick auf die Negative Dialektik wird auf die Verben „erschließen“ und „explizieren“ ebenso verwiesen wie auf die Idee der bestimmten Negation. ${ }^{22}$ Ein angeführtes Zitat aus „Traditionelle und kritische Theorie“ gibt vielleicht Aufschluss auf das Denkmuster, mit dem Müller-Doohm und Yos zu operieren scheinen: in der Textstelle besteht Horkheimer auf die Fortsetzung von theoretischer Anstrengung; ${ }^{23}$ und Müller-Doohm und Yos folgern, hierdurch werde meine Lesart ausgeschlossen. Diese Folgerung erscheint aber nur vor dem Hintergrund einer bestimmten impliziten Annahme berechtigt zu sein. Diese Annahme kann zwei Formen annehmen:

(A) Alle theoretischen Anstrengungen bestehen in einem Begründungsprogramm.

(B) Alle theoretischen Anstrengungen bestehen entweder in einem Begründungsprogramm oder beruhen darauf.

Wäre eine der beiden Annahmen wahr, wäre es in der Tat der Fall, dass ein positiver Bezug auf Selbstbesinnung, Erschließen, Explizieren oder bestimmte Negation ausreichte, um auf die Bejahung der Idee (und Notwendigkeit) eines Begründungsprogramms zu schließen. Aber warum sollen wir (A) oder (B) als wahr anerkennen? (A) verwischt wichtige Unterschiede zwischen verschiedenen theoretischen Anstrengungen (wie diejenigen zwischen Explizieren und Begründen, die ja durchaus auseinanderfallen können, etwa wenn jemand etwas expliziert, was er/sie für unbegründet hält). Und (B) bedürfte angesichts meiner Argumentationsnotizen gegen die Möglichkeit eines Begründungsprogramms einer argumentativen Untermauerung, auf deren Fehlen ich schon hingewiesen habe. Auch fehlen Belege dafür, dass Horkheimer und Adorno (A) oder (B) akzeptieren (wie ich unten noch im Detail darlege).

Eigentlich sollte dies schon ausreichen, um den Vorwurf des Zurechtlegens zurückzuweisen, aber ich will nun auch noch einzeln auf bestimmte Textpassagen eingehen:

$22 \mathrm{Rpl}, 7-8$.

23 TKT, 207, zit. in Rpl, 5. 
- Ich stimme Müller-Doohm und Yos darin zu, dass Kritik „nicht identisch mit ihrem Gegenstand“ ist. ${ }^{24}$ Es handelte sich nicht um Kritik, wenn sie ihren Gegenstand nur treu wiederspiegelte. (Als einen Versuch, eine solche Wiederspiegelung zu generieren, hat Horkheimer an der genannten Stelle die politische Ökonomie charakterisiert.) Aber dies allein reicht nicht aus, um sich einem Begründungsprogramm zu verschreiben (oder Horkheimer auf ein solches zu verpflichten). Kritik kann über ihren Gegenstand hinausweisen - z. B. dahingehend, dass sie auf seine (radikale) Veränderung drängt -, ohne deshalb ein Begründungsprogramm zu benötigen. Hier ist wieder das schon erwähnte Denkmuster am Werk: Wenn man einfach voraussetzt, dass Kritik ein solches Programm benötigt, dann können wir nur verstehen, wie Kritik vom Gegenstand unterschieden werden kann - also nur verstehen, wie sie Kritik sein kann -, wenn auch ein solches Programm vorliegt oder (wie implizit auch immer) angestrebt wird.

- Müller-Doohm und Yos betonen ganz richtig, dass Horkheimer auf den geschichtlichen Augenblick reagiert und seine Theorie auf Grund dessen angepasst hat. ${ }^{25}$ Sie sehen auch ganz richtig, dass Adorno dies ähnlich gesehen hat, ${ }^{26}$ und dass beide (Horkheimer und Adorno) davon überzeugt waren, dass Wahrheit einen Zeitkern habe, ${ }^{27}$ und sich daher Theorie, wenn sie wahr bleiben soll, selbst wandeln müsse. Sie übersehen jedoch, dass die Zeitkernthese gegen die Möglichkeit des von ihnen gewünschten Begründungsprogramms spricht (das ja eine Absicherung über den geschichtlichen Augenblick hinaus leisten soll) - und auch dagegen, anzunehmen, es bedürfte in jedem geschichtlichen Augenblick eines solchen Programms. Diese Textstellen sprechen daher letztlich für meine Interpretation, die Horkheimer und Adorno als Vertreter eines historischen Kontextualismus begreift, nicht für die Annahme, sie stünden für die gleichbleibende Notwendigkeit eines Begründungsprogramms. Darüber hinaus übersehen Müller-Doohm und Yos auch, dass für Horkheimer nicht einfach jeder Wandel angemessen ist, auch wenn er darauf insistiert, dass sich Theorie der historischen Situation anpassen müsse. Insbesondere gilt es, nicht zu verkennen, dass „,bei allem Wandel der Gesellschaft doch ihre grundlegende Struktur, das Klassenverhältnis in seiner einfachsten Gestalt, und damit auch die Idee seiner Aufhebung, iden-

24 Dieses Zitat stammt aus Horkheimer (1988b) (im Folgenden zit. als Ntg), 219 (zit. in Rpl, 4).

$25 \mathrm{Rpl}, 4$.

$26 \mathrm{Rpl}, 13$.

$27 \mathrm{Rpl}, 6$. 
tisch bleibt““. ${ }^{28}$ Nicht jeder Transformationsprozess der Kritischen Theorie lässt am Ende Kritische Theorie übrig. Wenn beispielsweise methodologisch von vornherein ausgeschlossen wird, dass es einer radikalen Aufhebung der grundlegenden Struktur (und damit verbundenen Werte) bedarf, um die gesellschaftlichen Missstände zu beseitigen, ${ }^{29}$ dann ist es fragwürdig, ob dies noch als Kritische Theorie (in Horkheimers Sinn) zu begreifen ist.

- Dass die Dialektik der Aufklärung nicht als Begründungsprogramm gelesen werden muss (und auch nicht sollte), liegt nicht nur daran, dass ihre zentralen Begriffe (wie „Selbstbesinnung“) ein solches Programm keineswegs implizieren. Es liegt auch daran, dass „den tiefsitzenden Defiziten der Vernunft nachgehen“30 - wie Müller-Doohm und Yos Adornos und Horkheimers Unterfangen in diesem Buch scheinbar gegen meine Lesart charakterisieren bedeutet, anzuerkennen, dass die Vernunft nicht in der Lage ist, ein Begründungsprogramm zu liefern. In der Dialektik der Aufklärung heißt es nicht von ungefähr, dass es unmöglich ist, „,aus der Vernunft ein grundsätzliches Argument gegen den Mord vorzubringen“. ${ }^{31}$ In diesem Sinne ist es zwar richtig, dass Adorno und Horkheimer nicht „Aufklärung und kritisches Denken“ preisgeben, aber nicht, dass sie damit die Idee „seine[r] Begründung“ beibehalten. ${ }^{32}$ Teil ihres Aufklärens der Aufklärung ist es, deren Begründungsprogramm der Kritik zu unterziehen.

- Auch berücksichtigen Müller-Doohm und Yos nicht, dass wir vermeiden können, Horkheimer ein „Oszillieren zwischen Idealismus und Pragmatismus“ vorzuwerfen. ${ }^{33}$ Eine solche Zuschreibung ist ganz unnötig, wenn man nicht einfach voraussetzt, dass negative Formulierungen nicht für Kritik ausreichen, dass es immer einer Entwicklung und Plausibelmachung einer „präzise[n] Vorstellung“ davon bedarf, „was als das Gerechte in der Gesellschaft gedacht werden kann“. ${ }^{34}$ Dass es gute Gründe geben mag, Negativist zu sein, ${ }^{35}$ wird nicht in Betracht gezogen.

28 TKT, 208.

29 Vgl. Schaub (2015) zu Honneths neuester Theorieentwicklung. Ich charakterisiere die Transformationsprozesse der Kritischen Theorie anderswo als multiple Morphingsequenzen (vgl. Freyenhagen 2018).

$30 \mathrm{Rpl}, 7$.

31 DdA, 140.

$32 \mathrm{Rpl}, 7$.

$33 \mathrm{Rpl}, 5$.

$34 \mathrm{Rpl}, 5$.

35 Vgl. Freyenhagen (2013a), insbes. Einl. u. Kap. 8. 
- Ähnlich dogmatisch ist das Referat der Position Habermas' in Erkenntnis und Interesse, die ich vermeintlich „nicht zur Kenntnis“ genommen habe. ${ }^{36}$ Dass es mir um die Grundsatzfrage ging, ob ein Begründungsprogramm überhaupt möglich und notwendig ist, und nicht darum, die einzelnen Varianten im Detail durchzuspielen, zählt für Müller-Doohm und Yos nicht. Es hätte einer argumentativ fundierten Variante bedürft, die Grundsatzfrage anders als ich zu beantworten. Es werden aber keine Argumente für Habermas' Thesen ins Feld geführt, sondern diese werden präsentiert, als wären sie unbestreitbar wahr.

- Müller-Doohm und Yos sehen richtig, dass Adorno „die Möglichkeit moralischen Handelns keineswegs ausschließen“ wollte. ${ }^{37}$ Aber es bleibt unklar, warum dies ein Einwand gegen meine Lesart sein soll. Erneut scheint hier das Denkmuster an der Arbeit, dass meine Ablehnung der Möglichkeit des Begründungsprogramms die Ablehnung alles anderen bedeuten müsse, inklusive der Möglichkeit moralischen Handelns. Dass Adorno selbst sich gegen Begründung wendet und gleichzeitig am moralischen Handeln festhält, habe ich anderswo im Detail dargelegt, ${ }^{38}$ wird aber von Müller-Doohm und Yos ohne weiteres ausgeschlossen. Dabei stehe ich mit einer solchen Interpretation nicht alleine. Auch nach Thomas Khuranas Lesart, auf die sich Müller-Doohm und Yos berufen, ${ }^{39}$ ist die Rolle der Reflexion bei Adorno nicht die der Begründung der Normen, sondern deren Brechung..$^{40}$ Khurana und ich stimmen auch dahingehend überein, dass für Adorno durchaus der Impuls „die letzte Instanz für die Entscheidung, was ethisch geboten ist“ sein kann, entgegen dem, was Müller-Doohm und Yos behaupten ${ }^{41}$ - nur ist der Impuls hier eben nicht als Gegenteil der Reflexion verstanden, sondern mit ihr in einer Konstellation verbunden. Bei Khurana heißt es entsprechend, dass ,[d]er Impuls [...] sich in diesem Subjekt aufgrund der moralischen Situation ergibt oder einstellt“ und dann die Verwirklichung von Normen ,ins Werk setzt" “. ${ }^{42}$ In diesem Zusammenhang wirkt der (aktivistisch-voluntaristisch konnotierte) Dezisionismusvorwurf besonders fehl am Platz, denn ich betone ja gerade die situationsgeleitete, objektive und in gewissem Sinne

$36 \mathrm{Rpl}, 10$.

$37 \mathrm{Rpl}, 9$.

38 Freyenhagen (2013a), insbes. Kap. 5-7.

39 Rpl, 9, Fn. 42.

40 Khurana (2013), insbes. 75 u. 82.

$41 \mathrm{Rpl}, 9$.

42 Khurana (2013), 78 u. 82; Hervorh. im Orig. 
passive Dimension des moralischen Handelns bei Adorno. ${ }^{43}$ Meiner Auffassung nach ist Adorno ein Aristoteliker, für den die Moral heutzutage allein im „ungeschminkt materialistischen Motiv überlebt“ ${ }^{44}$ und kein Dezisionist oder Existentialist.

- Ähnlich steht es um die Kritik der Ausbeutung, die Müller-Doohm und Yos Horkheimer und Adorno zuschreiben. Kein textlicher Beleg wird dafür angeführt, dass es sich hierbei um ,eine moralisch motivierte Kritik“ handele, ${ }^{45}$ sondern dies wird einfach behauptet - und das trotz der Kontroversen darum, ob und inwiefern Marx (auf den sich Horkheimer und Adorno stützen) seine Ausbeutungskritik als eine moralische verstand. Auch der nächste Schritt wonach eine moralisch motivierte Kritik implizieren soll, dass sich Horkheimer und Adorno um die Entwicklung von „Grundlagen einer Moralphilosophie“ bemühten ${ }^{46}$ - steht einfach als Behauptung da. Nur wer schon von vornherein ausgeschlossen hat, dass das Moralische nicht unabhängig von einem Begründungsprogramm existieren kann, übersieht hier das Nonsequitur. Erneut wird, was eigentlich zur Debatte steht, einfach von Anfang an auf eine bestimmte Weise beantwortet.

- Die Zweiteilung in theoretisches Begründungsprogramm und Zeitdiagnose, in Begründung und Durchführung von Kritik, in Theoriebildung einerseits und öffentliche Intervention andererseits mag bei Habermas vorliegen, jedoch zeigt dies weder, dass diese Zweiteilung über die Generationen der Frankfurter Schule hinweg akzeptiert worden ist (und wird), ${ }^{47}$ noch, dass es ein Defizit meines Ansatzes ist, diese beiden Ebenen nicht kategorisch zu trennen. Ich beschränke mich hier darauf, mit Blick auf Horkheimer beispielhaft zu zeigen, dass diese Zweiteilung keineswegs als notwendig für Kritische Theorie angesehen werden muss. Bei Horkheimer ist die Trennung zwischen Wissenschaftler und Staatsbürger ein Merkmal der traditionellen Theorie, die sich mit „der Trennung von Denken und Handeln bescheidet“ und damit

43 Vgl. Freyenhagen (2013a), insbes. Kap. 7, 9 u. Anh.

44 ND, 358; vgl. Freyenhagen (2013a), insbes. Kap. 9.

$45 \mathrm{Rpl}, 11$.

46 Ebd.

47 Es ist beispielsweise nicht klar, ob Honneth diese Zweiteilung akzeptiert. In seinem 2017 erschienenen Aufsatz zum emanzipatorischen Interesse schreibt Honneth: „In my view, it is indeed the case that critical theory is nothing but the continuation, by means of a controlled scientific methodology, of the cognitive labor that oppressed groups have to perform in their everyday struggles when they work to de-naturalize hegemonic patterns of interpretation and to expose the interests by which these are motivated“ (Honneth 2017, 919; meine Hervorh.). 
„auf die Humanität schon verzichtet“ hat. ${ }^{48}$ Dagegen konzipiert er die Kritische Theorie als Teil des „Kampf[es], zu dem sein Denken gehört, nicht das Denken als etwas Selbständiges, davon zu Trennendes“. 49

Es steht Müller-Doohm und Yos natürlich frei, zu argumentieren, dass Horkheimer falsch lag. Dies hätten sie auch mit Bezug auf die von mir vorgebrachten Textstellen tun können. Zum Beispiel hätten sie Horkheimers Ablehnung der „Annahme eines absoluten, übergeschichtlichen Subjekts“, der These von der „Auswechselbarkeit der Subjekte“ und der Möglichkeit von Neutralität anfechten können. ${ }^{50}$ Oder sie hätten kritisieren können, dass Horkheimer das Interesse an Umwandlung - nicht das Begründungsprogramm - als die einzige „spezifische Instanz" der Kritischen Theorie einsetzt. ${ }^{51}$ Aber nichts dergleichen findet sich in der Replik. Stattdessen sind wir mit einer von Dogmatismus gekennzeichneten Zurechtlegung von Texten konfrontiert.

\section{Kein Allheilmittel}

Dogmatisch muten auch manche der Einwände an, die nicht textlicher Natur sind.

Dass wegen der Nennung des Partisanenbegriffs gleich Carl Schmitt ins Spiel gebracht wird (und damit mein Ansatz in Verruf gebracht werden soll), ${ }^{52}$ hätte ich mir denken und mich dementsprechend davon distanzieren sollen. Ich habe wohl gehofft, es sei eindeutig genug, dass meine Idee des Kritischen Theoretikers als Partisan nicht - wie Müller-Doohm und Yos es von Schmitts Konzeption des Partisanen berichten - ,ganz bewusst neutral gegenüber jedweder Weltanschauung gehalten wurde““.53 Schließlich hatte ich das Interesse an der Emanzipation des Menschen aus versklavenden Verhältnissen als konstitutiv für (orthodoxe) Kritische Theorie (und damit Kritische Theoretiker) dargestellt - als etwas, was

48 TKT, 183 u. 216.

49 TKT, 190. Selbst wenn eine scharfe Trennung abgelehnt wird (wie bei Horkheimer), bedeutet dies nicht, dass nicht in bestimmten Texten die Selbstreflexion über Theorie im Vordergrund stehen kann (wie beispielsweise in TKT). Entscheidend ist, dass die Zeitdiagnose nicht kategorial von Theoriebildung abgetrennt wird und dass jedes theoretische Unterfangen selbst bereits als politisch angesehen wird, nicht erst die öffentliche Intervention des Intellektuellen.

50 TKT, 213-214 u. 196.

51 TKT, 216.

$52 \mathrm{Rpl}, 11-12$.

$53 \mathrm{Rpl}, 11$. 
allem anderen, ja sogar jeglichen Begründungsbemühungen vorausgeht. Nicht formale Kriterien, sondern eine substantielle Ausrichtung sind also für das Partisanentum der Kritischen Theorie bestimmend.

Es ist aber nicht nur so, dass meine Position nicht die von Schmitt (oder der Linksschmittianer) ist. Selbst die Art und Weise, auf die der Einwand vorgetragen wird, verrät ein unreflektiertes Verhältnis zum Begründungsprogramm. Meine Ablehnung desselben setze mich, so Müller-Doohm und Yos, besonderen Gefahren aus, nämlich der „Gefahr beliebiger Folgen“ und der „Willkür der Situation“ mit ihren „unberechenbaren Kräfteverhältnissen“. ${ }^{54}$ Suchen diese Gefahren nur oder besonders diejenigen heim, die sich dem Begründungsprogramm verweigern? Dies scheint mir - besonders angesichts der Erfahrungen aus der deutschen Geschichte des 20. Jahrhunderts - nicht der Fall zu sein. Selbst Kants Ethik ist, trotz seines (versuchten) Begründungsprogramms, vor Missbrauch, beliebigen Folgen und unberechenbaren Kräfteverhältnissen nicht gefeit geblieben. Trotz ihrer Hochachtung für Kant bleibt es etwa ein Verdienst Hannah Arendts, sich dieser Problematik gestellt zu haben. Müller-Doohm und Yos tun dies nicht.

Auch die Gefahr, dass der Vernunftmaßstab vage wird, verbinden MüllerDoohm und Yos mit dem Verzicht auf ein Begründungsprogramm. ${ }^{55}$ Dies zeugt zum einen erneut davon, dass die Möglichkeit dieses Programms schlicht vorausgesetzt wird; zum anderen wird wieder deutlich, dass dieses als eine Art Allheilmittel verstanden wird. Es scheint mir allerdings mehr als fragwürdig, ob etwa Kants kategorischer Imperativ als Vernunftmaßstab (trotz Begründungsprogramm) der Vagheit entkommt. ${ }^{56}$ Zudem wird nicht in Betracht gezogen, dass Horkheimers (und Adornos) Vernunftbegriff auch ohne Begründungsprogramm durchaus klar gefasst werden kann. ${ }^{57}$

Verwunderlich soll es sein, woher ich das emanzipatorische Interesse nähme, „wenn nicht aus dem Rückgriff auf ,übergeschichtliche Subjekte oder Reaktionsweisen“". ${ }^{58}$ Wieso dieses Interesse nicht aus Negativerfahrungen historisch situierter Menschen „genommen“ werden kann, verraten uns Müller-Doohm und Yos nicht. Warum kann es nicht, wie es mit Blick auf das Ziel der Kritischen Theorie bei Horkheimer heißt, in der „Not der Gegenwart“ gründen?5 ${ }^{59}$ Spricht nicht, um es mit Adorno zu sagen, Weh „Vergeh“? ${ }^{60}$ Müller-Doohm und Yos geben

$54 \mathrm{Rpl}, 12$.

$55 \mathrm{Rpl}, 3$.

56 Vgl. Freyenhagen (2011).

57 Vgl. Schiller (2018); vgl. Freyenhagen (o. J.).

$58 \mathrm{Rpl}, 3$.

59 TKT, 190.

60 ND, 203. 
hierauf keine Antwort, aber vermutlich steht erneut das Denkmuster dahinter, demzufolge uns ohne ein Begründungsprogramm alles in den Händen zerrinnt auch das emanzipatorische Interesse. Ich nehme an, sie gestünden zu, dass Menschen, die Ausbeutung, Versklavung und Elend ausgesetzt werden, ein Interesse daran entwickeln, diesen Umständen zu entfliehen. Aber es fehlt ihnen wohl die Absicherung, dass dieses Interesse auch wirklich emanzipatorisch ist. Und wahrlich sind nicht alle Reaktionen auf Negativerfahrungen emanzipatorisch. Nach Horkheimers und Adornos Analyse ist beispielsweise der Antisemitismus eine nicht zufällige Reaktion auf Negativerfahrungen mit dem, was wir als Zivilisation bezeichnen. ${ }^{61}$ Es gibt also in Bezug auf Negativerfahrungen ein wirkliches Problem. Wenn jedoch eine begründungstheoretische Absicherung nicht möglich ist, dann bleiben uns eben nur die anderen Mittel der Reflexion und Selbstdistanzierung, die ich schon nannte. Diese bieten uns keine Garantien, aber wenn ich mit der Ablehnung des Begründungsprogramms richtig liege, bietet uns nichts und niemand Garantien; somit fehlt auch nichts in dem Verständnis der Kritischen Theorie, das ich den LeserInnen ans Herz gelegt habe.

\section{Zum Schluss}

Die Pointe meines ursprünglichen Aufsatzes bestand darin, Lukács' OrthodoxieFrage in Bezug auf die Kritische Theorie aufzuwerfen, um sie dann aber anders zu beantworten, als es Lukács mit Blick auf den Marxismus tat. Nicht in der Methode liegt die Quintessenz - weder im historischen Materialismus (wie Lukács es auf den Marxismus bezogen meint) noch im Begründungsprogramm -, sondern in einem bestimmten substantiellen Interesse. Mit meinen Argumentationsskizzen wollte ich gleichzeitig darlegen, dass es eben nicht unkritisch ist, die Idee und Notwendigkeit des Begründungsprogramms abzulehnen. Stattdessen wird es dem kritischen Geist gerechter, diesem tiefsitzenden Defizit der Vernunft nachzugehen und zu erkennen, dass wir ohne Garantie, ohne absolute Absicherung in unseren kritischen Unternehmungen fortschreiten müssen.

Müller-Doohm und Yos haben darin nur Dogmatismus sehen können und mir wohl deshalb Dezisionismus vorgeworfen (denn wie anders als voluntaristisch und damit beliebig solle man zwischen Dogmen wählen?). Aber ihre Replik setzt einfach die Möglichkeit und Notwendigkeit des Begründungsprogramms voraus, die ja eigentlich in Frage gestellt wurden. Wenn hier also etwas als dogmatisch

61 Vgl. Freyenhagen (2019). 
zu bezeichnen ist, dann ihr starres Beharren auf dieser Möglichkeit und Notwendigkeit, ihr unkritisches Festhalten daran. Es brauchte, aber fehlte in Ihrer Replik an (überzeugenden) Gegenargumenten um meiner vorgeschlagenen Kehrtwende zu einer Kritischen Theorie jenseits des Begründungsprogramms als dogmatisch zu verwerfen.

Vielleicht ging es Müller-Doohm und Yos nur um Klärung, wie Kritische Theorie jenseits des Begründungsprogramms durchzuführen sei, was dann unter Selbstreflexion zu verstehen sei, und wie sich dabei Theorie und Praxis aufeinander beziehen sollen. Dies sind wichtige Fragen, aber sie wurden von ihnen zu sehr im Banne des Begründungsprogramms vorgetragen, um mich in dieser Replik einfach diesen Fragen zu widmen. An diesen Fragen müssen wir - gerne gemeinsam - weiterarbeiten, aber ein wichtiger erster Schritt ist es, den genannten Bann zu brechen. Es ist schon schwierig genug, die modernen Gesellschaften, ihre Krisen, Pathologien und Gerechtigkeitsdefizite einer kritischen Analyse zu unterziehen und damit zu Emanzipationskämpfen beizutragen, ohne es an der nötigen Selbstreflexion fehlen zu lassen. Auf diese Aufgabe sollten wir uns konzentrieren, statt nach unmöglichen Fundamenten $\mathrm{zu}$ buddeln und uns letztlich selber damit nur zu untergraben. ${ }^{62}$

\section{Literatur}

Adorno, T. W. (1997a), Minima Moralia [1951] (= Gesammelte Schriften 4), Frankfurt am Main. Adorno, T. W. (1997b), Negative Dialektik [1966] (= Gesammelte Schriften 6), Frankfurt am Main [ND].

Freyenhagen, F. (2011), Empty, Useless, and Dangerous? Recent Kantian Replies to the Empty Formalism Objection, in: Bulletin of the Hegel Society 63, 95-118.

Freyenhagen, F. (2013a), Adorno's Practical Philosophy: Living Less Wrongly, Cambridge.

Freyenhagen, F. (2013b), Ethical (Self-)Critique, in: Proceedings of the Aristotelian Society 113.3, 253-268.

Freyenhagen, F. (2015), Honneth on Social Pathology: A Critique, in: Critical Horizons 16.2, 131-152.

Freyenhagen, F. (2017), Was ist orthodoxe Kritische Theorie?, in: Deutsche Zeitschrift für Philosophie 65.3, 456-469, auch in: Danneman, R., Pickford, H., u. Schiller, H.-E. (Hg.) (2018), Der aufrechte Gang im windschiefen Kapitalismus. Modelle kritischen Denkens, Wiesbaden, 141-152.

62 Meinen Dank an Stefan Müller-Doohm und Roman Yos für die Replik; an Polona Curk, Christine Freyenhagen, Timo Jütten, Thomas Khurana, und Jörg Schaub für Kritik, bestimmte Formulierungen und andere Anregungen; und an die HerausgeberInnen für die Möglichkeit, auf die Replik zu antworten. 
Freyenhagen, F. (2018), Critical Theory: Self-Reflexive Theorizing and Struggles for Emancipation, in: Oxford Research Encyclopedia of Politics, URL: http://politics.oxfordre. com/view/10.1093/acrefore/9780190228637.001.0001/acrefore-9780190228637-e-195 (12.1.2019).

Freyenhagen, F. (2019), Adorno (and Horkheimer) on Antisemitism, in: Gordon, P., Hammer, E., u. Pensky, M. A., Blackwell Companion to Adorno, Oxford, \#\#\#SEITEN\#\#\#.

Freyenhagen, F. (o. J.), Objective reason and irrational society: the „linguistic turn“ in Horkheimer and Adorno, unveröff. Ms.

Honneth, A. (2017), Is there an emancipatory interest? An attempt to answer critical theory's most fundamental question, in: European Journal of Philosophy 25, 908-920, URL: https:// doi.org/10.1111/ejop.12321 (12.1.2019).

Horkheimer, M. (1988a), Traditionelle und kritische Theorie [1937], in: Gesammelte Schriften 4, Frankfurt am Main, 162-216 [TKT].

Horkheimer, M. (1988b), Nachtrag zu Traditionelle und kritische Theorie [1937], in: Gesammelte Schriften 4, Frankfurt am Main, 217-225 [Ntg].

Horkheimer, M., u. Adorno, T. W. (1997), Dialektik der Aufklärung. Philosophische Fragmente [1944] (= Adorno, T. W., Gesammelte Schriften 3), Frankfurt am Main [DdA].

Khurana, T. (2013), Impulse und Reflexion. Aporien der Moralphilosophie und die Moral der Aporien nach Adorno, in: Zeitschrift für kritische Theorie 19.36-37, 60-82.

Martin, W. (2009), Ought but Cannot, Proceedings of the Aristotelian Society 109, 103-128.

Müller-Doohm, S., u. Yos, R. (2018), Fatale Orthodoxie. Kritische Theorie auf der schiefen Bahn des Dezionismus. Eine Replik auf Fabian Freyenhagen, in: Deutsche Zeitschrift für Philosophie \#\#\#, \#\# [Rpl].

Schaub, J. (2015), Misdevelopments, Pathologies, and Normative Revolutions: Normative Reconstruction as Method of Critical Theory, in: Critical Horizons 16.2, 107-130.

Schiller, H.-E. (2018), Antagonismus. 12 Thesen zu Vernunft und Unvernunft in gesellschaftskritischer Perspektive, in: Danneman, R., Pickford, H., u. Schiller, H.-E. (Hg.), Der aufrechte Gang im windschiefen Kapitalismus. Modelle kritischen Denkens, Wiesbaden, 123-139. 